\title{
Original
}

CODEN-JHTBFF, ISSN 1341-7649

\section{Micro-CT Analysis of Rabbit Cancellous Bone Around Implants}

\author{
Yasuko Numata' ${ }^{1)}$, Toshiro Sakae², Hiroshi Nakada ${ }^{3)}$, Taketoshi Suwa ${ }^{3)}$, Racquel Z. LeGeros ${ }^{4)}$, \\ Yoshimitsu Okazaki ${ }^{5}$ and Kihei Kobayashi' ${ }^{6}$
}

\author{
1) Nihon University Graduate School of Dentistry at Matsudo, Complete Denture Prosthodontics, Chiba, Japan \\ 2) Department of Histology, Cytology, and Developmental Anatomy, Nihon University School of Dentistry at Matsudo, Chiba, Japan \\ 3) Department of Gnatho-Oral Prosthetic Rehabilitation, Nihon University School of Dentistry at Matsudo, Chiba, Japan \\ 4) Department of Biomaterials and Biomimetics, New York University College of Dentistry, New York, 10010, USA \\ 5) Institute for Human Science and Biomedical Engineering, National Institute of Advanced Industrial Science and Technology, Ibaraki, \\ Japan \\ ${ }^{6)}$ Nihon University Research Center, Chiba, Japan \\ (Accepted for publication, May 31, 2007)
}

\begin{abstract}
The aim of this study is to observe the three-dimensional structure of cancellous bone formed around dental implants using micro-CT.Methods: Ti implants (Ti-15\%Zr-4\%Nb-4\%Ta alloy, 2.8mm diameter, 10mm length, machine-polished) were placed in surgically created bone defects in rabbit femurs (New Zealand White Rabbits). The rabbit femurs with the implants were removed 6 weeks after implantation and preserved in formalin. Micro-CT analysis was performed using three types of machine, TOSCANER-31300 mhd ${ }^{\circledR}$ (Voltage: 80 kV, Current: 120 ìA, Focus size: 45ìm, Toshiba Co., Japan), TDM1000 ${ }^{\circledR}$ (Voltage: 95 kV, Current: 6 ìA, Focus size: 5 ìm, Yamato Kagaku, Japan) and SkyScan-2011 ${ }^{\circledR}$ (Voltage: 80 kV, Current: 200 ìA, Focus size: 0.4ìm, Tohken Co., Japan) and data obtained were compared. The best conditions were taken to obtain the best images for each equipment.Results: Critical conditions for micro-CT analyses included: slice pitch, slice thickness, voltage, current and type of equipment. The smaller the slice spacing and the smaller the slice thickness, the clearer the micro-CT images obtained. Many artifacts appeared with TOSCANER-31300 mhd ${ }^{\circledR}$, but disappeared with TDM1000 ${ }^{\circledR}$. SkyScan-2011 ${ }^{\circledR}$ provided the most detailed images. Micro-CT made possible quantitative analysis of newly formed bone surrounding the dental implants. Conclusion: Micro-CT analysis is a good non-destructive method to obtain three-dimensional structure of trabecular bone with maximum resolution (10 ìm).
\end{abstract}

Key words: Micro-CT, Bone, Implant, Artifact

\section{Introduction}

Micro-computed tomography (micro-CT) is a non-destructive method for analysis bone microstructure ${ }^{1)}$. This method provides a three dimensional reconstruction image with the possibility of easily obtaining information on morphology and bone density from the CT-values. Information on bone architecture from micro-CT analysis was shown to be related to the mechanical properties of bone $^{2)}$. Development of high-resolution micro-CT has remarkably advanced in recent years. However, artifact images have been observed when micro-CT is used on specimens with metals ${ }^{3)}$, as in the case of dentam implants. For this reason, detailed analysis of bone surrounding and/or attached to the implant had not been carried out successfully. The purpose of this study was to compare the images obtained using three types of micro-CT equipment

Correspondence to:Dr. Yasuko Numata, Nihon University Graduate School of Dentistry at Matsudo, Complete Denture Prosthodontics.2-870-1 Sakaecho Nishi Matsudo city Chiba 271-8587, tel: 047-360-9379 fax: 047-360-9376, e-mail: numata.yasuko@nihon-u.ac.jp with different optimum scan modes and imaging-processes and determine the most efficient system that will provide images of the implant-bone interface with the highest resolution and reduced metal artifact .

\section{Materials and Methods}

\section{Laboratory animals}

New Zealand White male rabbits (sixteen weeks old) were used in this study.

\section{Implants}

Ti-15\%Zr-4\%Nb-4\%Ta alloy cylindrical implants, $2.8 \mathrm{~mm}$ in diameter and $10 \mathrm{~mm}$ in length were implanted in the femur and tibia.

\section{Operation}

The animal experiment protocol for this study was approved by the Ethical Committee for Animal Experiments, School of Dentistry at Matsudo (No. ECA-03-0003). General anesthesia was 
Yasuko Numata et al.: Micro-CT analysis of rabbit bone around implants

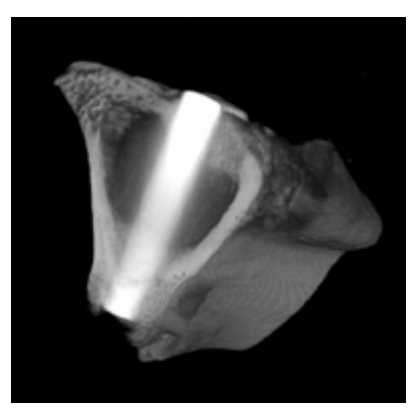

Fig. 1 Micro-CT image obtained using TOSCANER ${ }^{\circledR}$. Note: bone structure was clearly shown, but artifact image was also present.

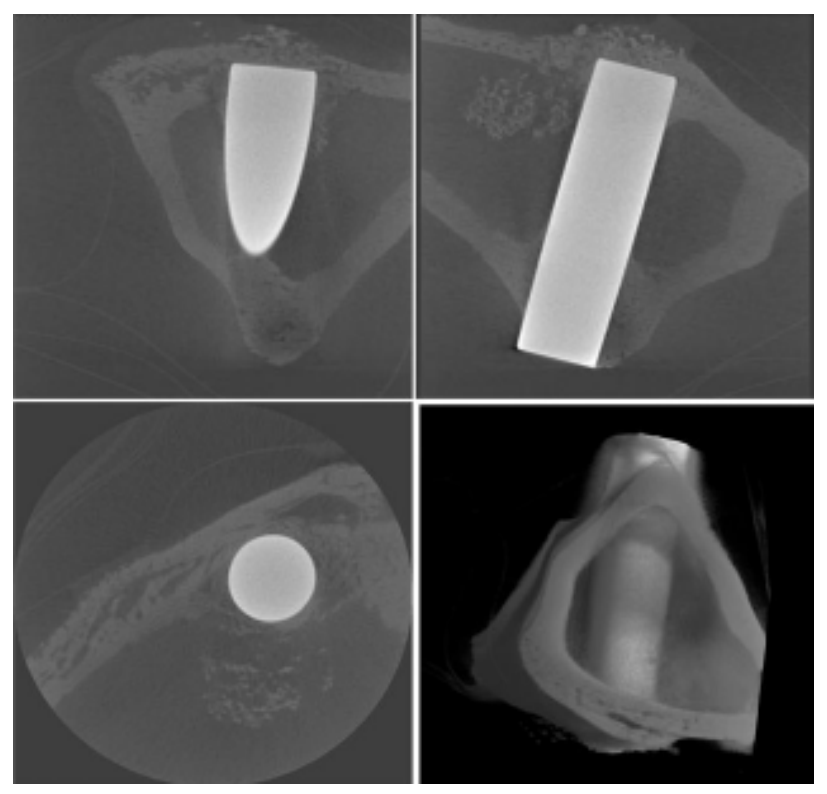

Fig. 2 Micro-CT images lobtained using TDM1000 ${ }^{\circledR}$. Note: artifact image due to the Ti implant was greatly reduced.

performed by injection of pentobarbital sodium (Nembutal ${ }^{\mathrm{o}}$, Dainippon Pharmaceutical Co., Ltd., Japan) via the auricular vein at a rate of $25 \mathrm{mg} / \mathrm{kg}$. Subsequently, an incision was made in the apophysis on the central side of the femur or the tibia, and the bone surface was exposed. An implantation pit was made in parallel to the long axis of the femur using an engine for implants (Implanter-S ${ }^{\circledR}$, Kyocera Co., Japan) at a low rotation rate (gear ratio, 1:16), and an implant was inserted. The rabbits were killed 6 weeks after implantation, and non-demineralized sections were prepared from the bones. For the implant pull-out test, the implant was pulled up in crosshead speed at $0.5 \mathrm{~mm} / \mathrm{min}$. with a small size desk test machine (EZ Test ${ }^{\circledR}$, SHIMADZU Co., Japan) ${ }^{2}$.

Observation by micro-CT: Three types of micro-CT were used in this study: TOSCANER-31300mhd ${ }^{\circledR}$ (Toshiba Co., Japan), TDM1000 ${ }^{\circledR}$ (Yamato Kagaku Co., Japan) and SkyScan-2011 ${ }^{\circledR}$ (Tohken Co., Japan). Image acquisition was performed by the cone beam scan method with the non-helical mode, image acquisition normal to bone long axis. As for the experimental conditions other than these, the best settings were selected for each device (Table I). CT slice images were then reconfigured for
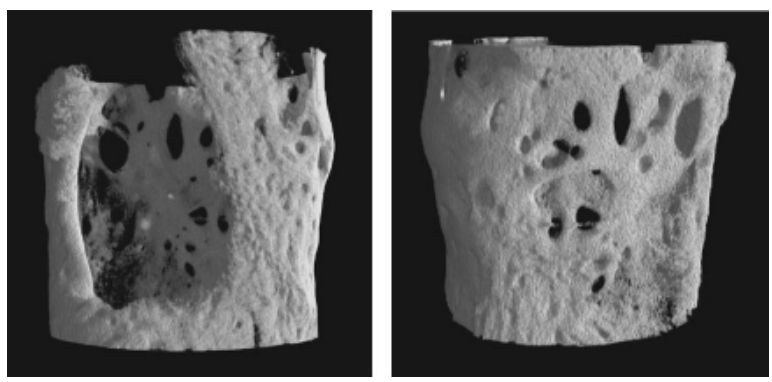

Fig. 3 Micro-CT images obtained using SkyScan ${ }^{\circledR}$. Note: Ti-implant was pulled out, and fine structure of bone was observed.

three dimensional images by three-dimensional trabecular structure measurement software (3D BON ${ }^{\circledR}$, RATOC System Engineering, Japan) and structure of bone was analyzed.

\section{Results}

In the images obtained using TOSCANER-31300mhd ${ }^{\circledR}$, structure of the cancellous bone was clearly observed in high definition with maximum resolution of $10 \mu \mathrm{m}$ (Fig. 1). However, the implant caused the appearance of artifact images. In the images obtained using TDM $1000^{\circledR}$, artifacts around the implants were considerably reduced by setting the best viewing conditions (Fig. 2). Reduction of artifact resulted in a clear discrimination of attached bone from the Ti alloy implant. In images obtained using SkyScan-2011 ${ }^{\circledR}$, microstructure of bone trabecula were observed in super high definition with maximum resolution, $150 \mathrm{~nm}$ (Fig. 3). In this case, the fine structure of the bone surface could also be observed.

\section{Discussion}

Micro-CT is a non-destructive analytical method for investigating bone structure ${ }^{1,4-8}$. This method, unlike the conventional light microscope method, does not need tedious specimen preparation and specialized skills in microscopy. With recent micro-CT technology, it is possible to reduce the artifact that disturbs accurate observation of the newly formed bone around and/or attached to the implants. In addition, a remarkable improvement of the picture was realized ${ }^{8-10)}$ and successful development of 0.4 ìm focus size was obtained using SkyScan$2011^{\circledR}$. Maximum resolution (150 nm) was obtained by adding more improvements to the detector and the sample stage. The resolution obtained in our study $(150 \mathrm{~nm})$ is greater than the higher resolution $(200 \mathrm{~nm})$ reported by other studies ${ }^{10,11)}$.

Table 1. Experimental conditions for the micro-CT observations.

\begin{tabular}{lccc} 
& TOSCANER & SkyScan-2011 & TDM1000 \\
\hline Voltage & $80 \mathrm{kv}$ & $80 \mathrm{kv}$ & $95 \mathrm{kv}$ \\
Current & $120 \mu \mathrm{A}$ & $200 \mu \mathrm{A}$ & $6 \mu \mathrm{A}$ \\
Focus & $45 \mu \mathrm{m}$ & $0.4 \mu \mathrm{m}$ & $5 \mu \mathrm{m}$ \\
Slice thicness & $0.05 \mathrm{~mm}$ & $0.005 \mathrm{~mm}$ & $0.026 \mathrm{~mm}$ \\
\hline
\end{tabular}


Bone mass does not always directly equate with bone strength. Thus, bone mass information must be carefully considered when associating it with bone mechanical properties. The morphological and architectural properties of bone can be obtained from microCT analyses.

\section{Conclusions}

This study showed that it is possible to get micro-CT images with much higher resolution and reduced metal artifact than has been reported previously. Micro-CT is a useful tool in determining morphology and structure of the newly formed bone surrounding the implant or attached to the implant. Micro-CT is especially important in assessing the properties of the spongious or trabecular bone such as bone density, trabecular bone thickness and connectivity. These bone characteristics may influence bone strength. Micro-CT is a valuable analytical tool in assessing the implant-bone interface and the effect of implant surfaces on the quality and structure of the newly formed bone.

\section{Acknowledgements}

The authors gratefully acknowledge Toshiba Co., Japan, Yamato Kagaku, Japan, and Tohken Co., Japan for the use of the micro-CT equipment.

This study was supported in part by the Japan Society for the Promotion of Science: Grant-in-Aid for Scientific Research (C) (18592145).

\section{References}

1. Ito M.: Non-destructive assessment of bone quality using micro-computed tomography (micro-CT) and synchrotron radiation CT. Endocrinology and Diabetology 19:276-283, 2004

2. Borah B., Dufresne T.E., Chmielewski P.A., Gross G.J., Prenger
M.C. and Phipps R.J.: Risedronate preserves trabecular architecture and increases bone strength in vertebra of ovariectomized minipigs as measured by three-dimensional microcomputed tomography. J Bone Miner Res 17: 139-147, 2002

3. Webb S.: The Physics of Medical Imaging. Institute of Physics Publishing, Bristol and Philadelphia, 1993, Book

4. Machida T.: A Study of Osseointegration of Titanium Alloy Implants. Nihon Univ J Oral Sci 30:245-257, 2004•@

5. Beck T.J., Looker A.C., Ruff C.B., Sievanen H. and Wahner H.W.: Structural trends in the aging femoral neck and proximal shaft: analysis of the Third National Health and Nutrition Examination Survey dual-energy X-ray absorptiometry data. J Bone Miner Res 15:2297-2304, 2000

6. Adachi T.: Computational Evaluation of Mechanical Properties of Bone. Clinical Calcium 12:1556-1558, 2002

7. Mulder L., van Groningen L.B., Potgieser Y.A., Koolstra J.H. and van Eijden T.M.: Regional differences in architecture and mineralization of developing mandibular bone. Anat Rec A Discov Mol Cell Evol Biol 288:954-961, 2006

8. Genant H.K. and Jiang Y.: Advanced imaging assessment of bone quality. Ann N Y Acad Sci 1068:410-428, 2006

9. Carballido-Gamio J. and Majumdar S.: Clinical utility of microarchitecture measurements of trabecular bone. Curr Osteoporos Rep 4:64-70, 2006

10. Powitzky E.S., Hayman L.A., Chau J., Bartling S.H., Gupta R. and Shukla V.: High-resolution computed tomography of temporal bone: Part IV: Coronal postoperative anatomy. J Comput Assist Tomogr. 30:548-554, 2006

11. Hashimoto K., Kawashima S., Araki M., Iwai K., Sawada K. and Akiyama Y.: Comparison of image performance between cone-beam computed tomography for dental use and fourrow multidetector helical CT. J Oral Sci 48:27-34, 2006 